\title{
Pobreza y Desnutrición en el Perú: Explorando la Última Década
}

\author{
Luis Reyes Lostaunau
}

Resumen: En este artículo se aborda la relación existente entre pobreza y desnutrición, partiendo del importante concepto de Estado Nutricional Óptimo, que establece el equilibrio entre la ingestión de alimentos y los requerimientos nutricionales por un lado y, por el otro, permite visualizar las múltiples causas que influyen en el equilibrio, pero también en el desequilibrio generando malnutrición, que en el caso de la población pobre se expresa principal, aunque ahora ya no exclusivamente, como desnutrición.

Palabras claves: Estado nutricional óptimo, malnutrición, desnutrición crónica, pobreza calórica, pobreza extrema. 


\section{Luis Reyes Lostaunau}

La pobreza y la desnutrición son dos fenómenos multicausales que se retroalimentan, en la actualidad son el resultado de las políticas tanto económicas como sociales que se han venido aplicando en el país desde hace varios lustros, pero también son un punto de partida. En ese sentido, la extensión e intensidad de la pobreza y la desnutrición vigentes tienen que ver, también, con las vidas futuras de quienes la padecen, fenómeno que cuando se desplaza de lo individual a lo colectivo pone sobre el tapete la problemática del desarrollo futuro del país.

La palabra pobreza es una abstracción que sintetiza varias formas concretas de ser pobre; igualmente, el concepto de desnutrición se expresa en la realidad de diversas maneras. Como resultado de la íntima relación que existe entre las pobrezas y la desnutrición en sus diferentes manifestaciones aparecen múltiples grupos de personas, siendo el caso de los niños el más delicado, que se ven afectados por la combinación de estos flagelos.

La pobreza es estudiada desde la perspectiva de la Línea de Pobreza (LP), como total (PT), no extrema (PNE)y extrema (PE); mirada desde las Necesidades Básicas Insatisfechas (NBI) considera pobres a los que tienen una NBI e indigentes o en miseria a los que tienen dos o más NBI. Una tercera forma de acercarse es considerando la Pobreza Calórica (PC), que es el resultado del déficit de la ingestión de alimentos frente a los requerimientos de nutrientes de los seres humanos.

En relación a la desnutrición, ésta se expresa como crónica, global y aguda. La crónica se produce cuando hay un retraso de la talla de los niños o niñas respecto a la talla esperada para su edad, lo común es que los datos estén referidos a menores de 5 años aunque hay mediciones realizadas a niños y niñas de mayor edad; la global registra el déficit de peso en relación al peso esperado para la edad; y la aguda, establece el déficit de peso para la talla aun cuando ésta es menor que la esperada para la edad de los niños y niñas.

Tanto la pobreza como la desnutrición responden a múltiples causas y producen también múltiples efectos. Hay dos conceptos de pobreza que están relacionados con la desnutrición, son la PE y la PC. Antes de abordarlos, ubiquemos la importancia del estado nutricional óptimo 
Pobreza y desnutrición en el perú: explorando la última década

\section{Sobre el estado nutricional óptimo.}

En la figura 1 están registrados los diversos elementos que influyen en el establecimiento del equilibrio, o desequilibrio, entre la ingestión de alimentos y el requerimiento de nutrientes. El equilibrio, producirá el Estado Nutricional Óptimo que al igual que su antípoda no es un problema exclusivamente de alimentación. Esta percepción, ha sido el craso error que las políticas alimentarias, como parte de las políticas sociales, han cometido creyendo que la desnutrición se combatía aportando a los destinatarios sólo alimentos, que adquirían la forma, en el mejor de los casos, de preparaciones balanceadas.

Por el lado de la ingestión de alimentos tenemos: la capacidad de compra, que depende del nivel de ingreso familiar, y éste a su vez del tipo de empleo; la decisión de compra, que depende, a su vez, de la cultura, de la educación y del nivel de información que posea la familia; el manejo de alimentos en el hogar, que responde a los valores establecidos en el seno de la familia, por ejemplo que se privilegie la ración del jefe de hogar; finalmente, sin pretender agotar los factores que influyen en la ingestión de alimentos, las características del individuo que se expresan, concretamente, como estado de salud, talla, peso, edad, sexo, conducta alimentaria y aspecto afectivo.

Son varios los factores que intervienen en el requerimiento de nutrientes de las personas, entre otros se tiene: el mantenimiento orgánico, que depende de la actividad física que realiza la persona, a mayor gasto de energía mayor será el requerimiento de nutrientes, y del lugar de residencia, por ejemplo en la sierra se gasta más energía que en la costa. La fase de crecimiento en la que se encuentra el ser humano determina la cantidad de nutrientes requerida, en el caso de los niños de corta edad el requerimiento de energía por kilo de peso es hasta dos y tres veces la cantidad de energía que necesitan los adultos. Otros aspectos a considerar son, el estado de la salud, la edad, el sexo y la afectividad. Queda claro que el estado nutricional óptimo depende de múltiples factores que rebasan el mero ámbito de la alimentación. 
Figura $\mathrm{N}^{\circ}$ 1: Estado Nutricional Óptimo

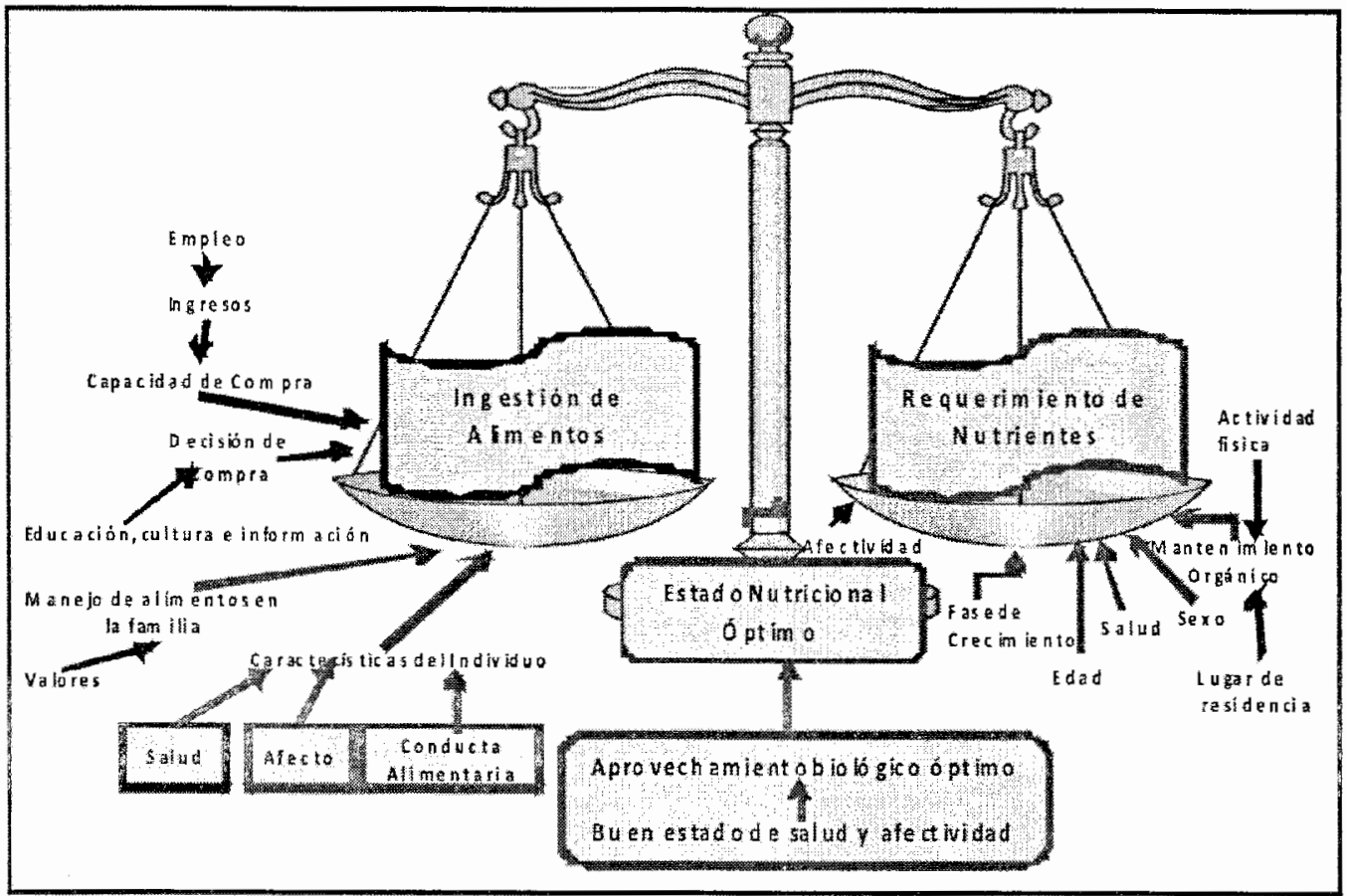

Nota: El original de esta figura ha sido tomado del libro de Maria Teresa Restrepo "Estado nutricional y crccimicnto fisico". Esta figura ha sido modificada por el autor del presente artíeulo.

\section{La desnutrición como una de las manifestaciones de la malnutrición.}

La alteración del estado nutricional óptimo, que es el desequilibrio que se produce entre la ingestión de alimentos y el requerimiento de nutrientes produce lo que se conoce como malnutrición, ésta no aparece intempestivamente, por el contrario atraviesa distintas fases antes de expresarse como desnutrición, carencia específica de algún nutriente, subnutrición o sobrenutrición.

En la desnutrición el consumo se ubica por debajo del requerimiento de nutrientes y se manifiesta como leve, moderada o severa dependiendo de la intensidad del desequilibrio; la carencia específica, como su nombre lo indica muestra la deficiencia relativa o absoluta de un nutriente, verbigracia el hierro, la vitamina $\mathrm{C}$ o la vitamina $\mathrm{A}$ que 


\section{Pobreza y desnutrición en el perú: explorando la última década}

causan, respectivamente, anemia ferropénica, escorbuto y ceguera nocturna; la subnutrición, donde la persona se adapta a la deficiencia crónica de nutrientes que por lo general responde a una deficiente ingestión de alimentos asociada a un deficiente aprovechamiento biológico de nutrientes, motivo por el cual no presenta manifestaciones de desnutrición. Es el caso de los niños que presentan retardo en el crecimiento y su peso se ha adecuado a su estatura; Finalmente, la sobrenutrición consiste en un aporte calórico superior al gasto energético, produciendo sobrepeso u obesidad, siendo este último el que requiere mayor cuidado. Hay que tener en cuenta que las personas pueden tener sobrepeso $\mathrm{u}$ obesidad, pero tener simultáneamente alguna carencia relativa. Estar gordito no es sinónimo necesariamente de estar bien nutrido. Tradicionalmente estos cuadros han sido característicos de personas con altos ingresos, pero en la actualidad hay gente pobre que sufre de sobrepeso u obesidad, ello responde a dietas que son influidas por la publicidad de alimentos que no constituyen la mejor elección. En adelante abordaremos los dos primeros casos orientados a distintos grupos etarios.

\section{Evolución de la desnutrición crónica en niños menores de 5 años.}

Durante la década 1996-2005, según la ENDES, la desnutrición crónica en niños menores de 5 años en el Perú se ha mantenido relativamente estable, de $25.8 \%$ pasó a $24.1 \%$. Se sabe que los promedios nacionales encubren grandes desigualdades entre las regiones (departamentos), para el año 1996 en Huancavelica la desnutrición crónica alcanzó al $50.3 \%$ de los niños menores de 5 años en tanto que en Lima y Moquegua fue $10.7 \%$ y $10.1 \%$ en Tacna. La medición del año 2000 arrojó 53.4\% para Huancavelica y en el polo opuesto $5.4 \%$ para Tacna. Además de las notorias desigualdades, podemos afirmar que son las regiones donde la pobreza ha estado más extendida donde la desnutrición crónica creció respecto a la medición anterior (Huancavelica, Huánuco, Cusco, Cajamarca, Puno, Ancash, Amazonas y Ucayali). Hay aspectos estructurales adversos que el modelo económico aplicado con gran intensidad desde finales de los años 80 no ha podido revertir, más aun cuando las políticas sociales fueron mal concebidas y peor administradas.

El gráfico 1 permite visualizar la probable trayectoria recorrida por los niños 


\section{Luis Reyes Lostaunau}

desnutridos crónicos a través del período 1996-2005. Observemos que en cada una de las ENDES el $80 \%$ de los niños medidos tenían edades que no permitían que hubieran sido medidos en la encuesta anterior, por la sencilla razón de que no habían nacido. Tal como se aprecia en el gráfico 1, solamente los niños que tuvieron 0 años en 1996, y posteriormente sus pares en el año 2000, participaron en la ENDES siguiente a través de una muestra, lo que significa que, en general, no fueron considerados los mismos niños en la medición por no ser una encuesta panel.

La ENDES Continua 2004-2005 establece que el 30\% de los niños y niñas de 5 años de edad sufren desnutrición crónica, aunque en el grupo de niños y niñas de hasta los 12 años de edad la desnutrición crónica desciende a alrededor del $28 \%$. En todo caso, lo que queda claro es que el porcentaje de desnutridos crónicos para las edades superiores al grupo etario de 0 a 4 años es mayor.

Gran preocupación produce, el que aproximadamente 3 de cada 10 niños y niñas de hasta 12 años de edad son desnutridos crónicos a nivel nacional según la ENDES Continua. En lo inmediato, resulta afectada su salud y su capacidad cognitiva, lo que tiene que ver directamente con el rendimiento escolar, en aquellos niños y niñas que están insertos al sistema educativo. La situación es más grave en las regiones donde la desnutrición crónica alcanza porcentajes más altos. Así, pausada pero de manera segura, se gesta la desigualdad de oportunidades en los niños y niñas que residen en espacios geográficos y económicos sociales nítidamente diferenciados.

La multicausalidad de la desnutrición y del deficiente desempeño educativo convierte en errada toda pretensión de "resolver" éstos problemas considerándolos como si fueran unicausales. Al agregar los aspectos tales como la subnutrición y la anemia, que afecta, esta última, al $46 \%$ de los niños menores de 5 años a nivel nacional y al $53 \%$ en el área rural, hace que la dimensión de la problemática presentada crezca, porque a más edad, ceteris paribus, mayor cantidad de niños y niñas presentan deficiencias nutricionales, con el consecuente déficit en el rendimiento escolar. 
Pobreza y desnutrición en el perú: explorando la última década

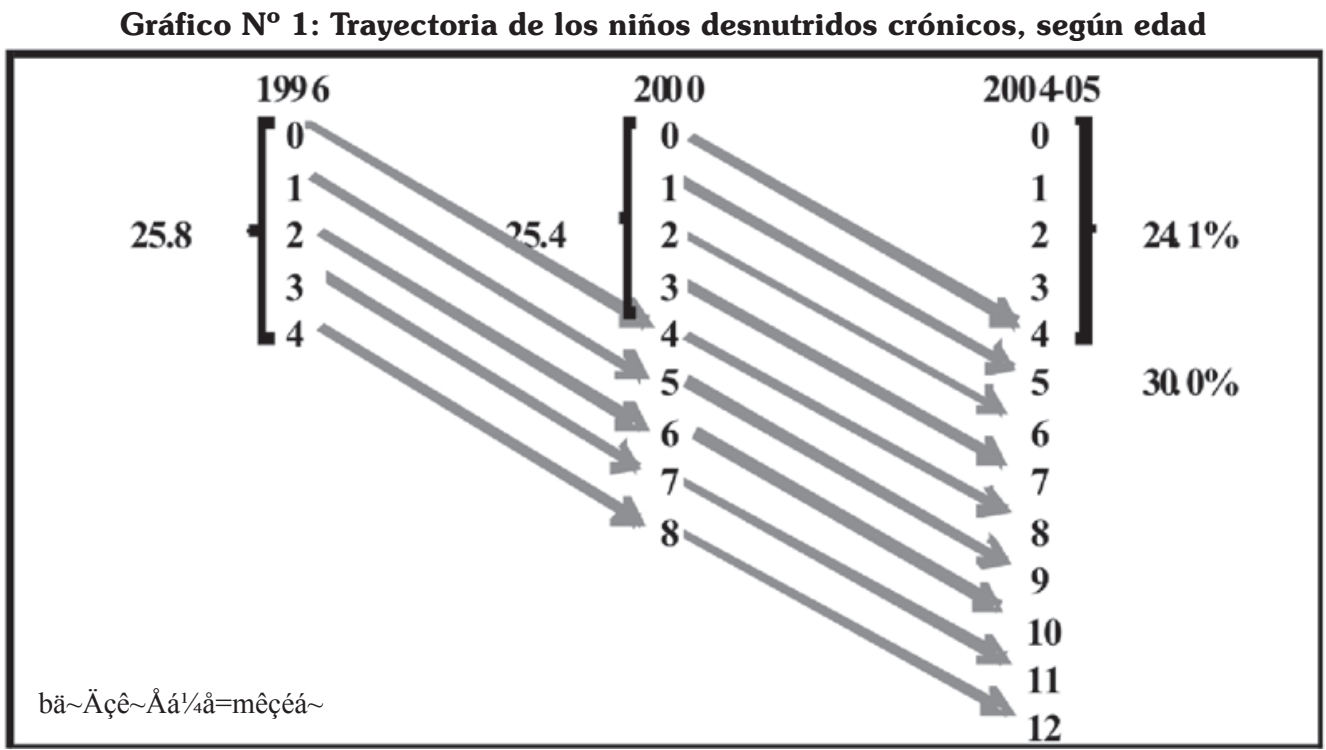

Lo que está ocurriendo con la niñez de nuestro país, invita a preguntarnos acerca de si es que se está construyendo un futuro con paz social. Porque así como el presente es la cosecha de lo que se sembró en el pretérito, la calidad de lo que se siembre en el presente es lo que determinará la calidad de la cosecha del futuro. Si se mantienen políticas de exclusiones cuantitativas y cualitativas en los campos de la economía, de la salud, del saber, entre otros, se estará construyendo una sociedad que carecerá de cohesión económica, social, política, con ausencia de una ciudadanía formalmente uniforme cuyos resultados serían, muy probablemente, muy nefastos para los niños, jóvenes y adultos que decidan vivir en el país. La población de hasta 12 años de edad desnutrida crónica según la ENDES Continua es cercana a los 2 millones. Son niños y niñas que dentro de pocos años serán adultos y formarán hogares sin haber desarrollado plenamente sus capacidades, quedando algunas de ellas truncas, contribuyendo a la transmisión intergeneracional de la pobreza.

La política multisectorial de exclusión tiene dos planos: el primero, el más obvio y burdo, es el que tiene que ver con la exclusión física de las personas en diversos ámbitos de los cuales podemos mencionar el empleo, la salud, la educación, la cultura, entre otros; y el segundo, el "sofisticado" propio del neoliberalismo en nuestros lares, donde 


\section{Luis Reyes Lostaunau}

las personas físicamente están "incluidas", aunque la calidad de los servicios que reciben es deficiente porque todos los aspectos, directos e indirectos, que tienen que ver con éstos han sido precarizados, pulverizando, así, todo intento de construcción de igualdad de oportunidades

\section{Pobreza, desnutrición y desarrollo}

Más allá de las historias de vida de las personas, donde la gran parte de ellas se ven imposibilitadas de superar sus adversidades y dependen de la voluntad y hasta del estado de ánimo de quienes deciden sobre el uso de los recursos públicos, está la salida colectiva, para lo cual se tiene, como diría Gerschenkron, que superar los obstáculos al desarrollo.

En ese sentido, los obstáculos se manifiestan concretamente como: pobreza; desnutrición; salud precaria; educación deficiente; corrupción, acompañada de la ausencia parcial o total de moral; estrechez de capitales, que no es superada por la acción firme del Estado que cuando obtiene recursos privilegia al sector privado nutriendo a la banca de segundo piso; deuda externa, que constituye un drenaje de capitales que de regresar lo hace en magnitud inferior; $y$, sin agotar todos los obstáculos, la dependencia tecnológica, ligada a la propiedad intelectual, donde los beneficios de los propietarios son vitalicios y se mantienen inclusive hasta post morten.

Estos obstáculos, fortalecen la tendencia contraria a la inclusión. Amplios sectores de la población han sido expulsados de los mercados más elementales, lo que no es bueno para el desarrollo del mercado interno. Cerca de la mitad de los peruanos son pobres y uno de cada cinco vive en pobreza extrema. Ante la firme decisión pública y privada de no hacer crecer el mercado interno es que persiste la ideología de orientar lo mejor de la actividad económica nacional a satisfacer las necesidades de los mercados externos, sin agrandar el mercado interno.

Exportar no es malo, lo malo es mantener un modelo primario exportador donde el azúcar y el algodón que antaño se exportaban, hoy son reemplazados por la 
exportación de mangos, uvas, paltas, espárragos, páprika, aceitunas, alcachofas, lúcumas, entre otros productos, que siguen manteniendo a los trabajadores agrícolas sumidos en la pobreza como se ha podido ver en el edén del pleno empleo, el sur chico. Las exportaciones tradicionales, conformada por productos pesqueros, agrícolas, minerales y petróleo, el año 2006 superaron el 77\% de las exportaciones totales. Las actividades primario exportadores se han fortalecido reprimarizando la economía del país.

Las grandes ciudades como Lima Metropolitana y Callao han visto crecer las actividades de servicios y comercio donde la rotación del empleo es veloz, donde el empleo seguro y de calidad es pequeño en relación a la oferta laboral.

\section{Sobre las pobrezas extrema y calórica y la desnutrición.}

Se considera pobres extremos a todas aquellas personas cuyos gastos per cápita no llegan a cubrir el costo de una Canasta Básica Alimentaria (CBA). Se encuentran en pobreza calórica las personas que tienen un consumo de calorías inferior a sus requerimientos calóricos. El consumo de calorías se contabiliza independientemente de si tuvieron por origen la compra, el autoconsumo, el pago en especies o la transferencia de instituciones públicas o privadas; se fijan los requerimientos calóricos considerando sólo el sexo y la edad de los individuos.

Analizando la información que aparece en el gráfico 2, observamos que la PC es mucho mayor que la PE tanto a nivel nacional como en el área urbana; en Lima Metropolitana y el Callao la distancia es mayúscula. En el campo las pobrezas extrema y calórica casi coinciden. 
Gráfico No 2: Perú: Pobreza extrema y pobreza calórica total y por áreas, 2004. (cifras relativas)

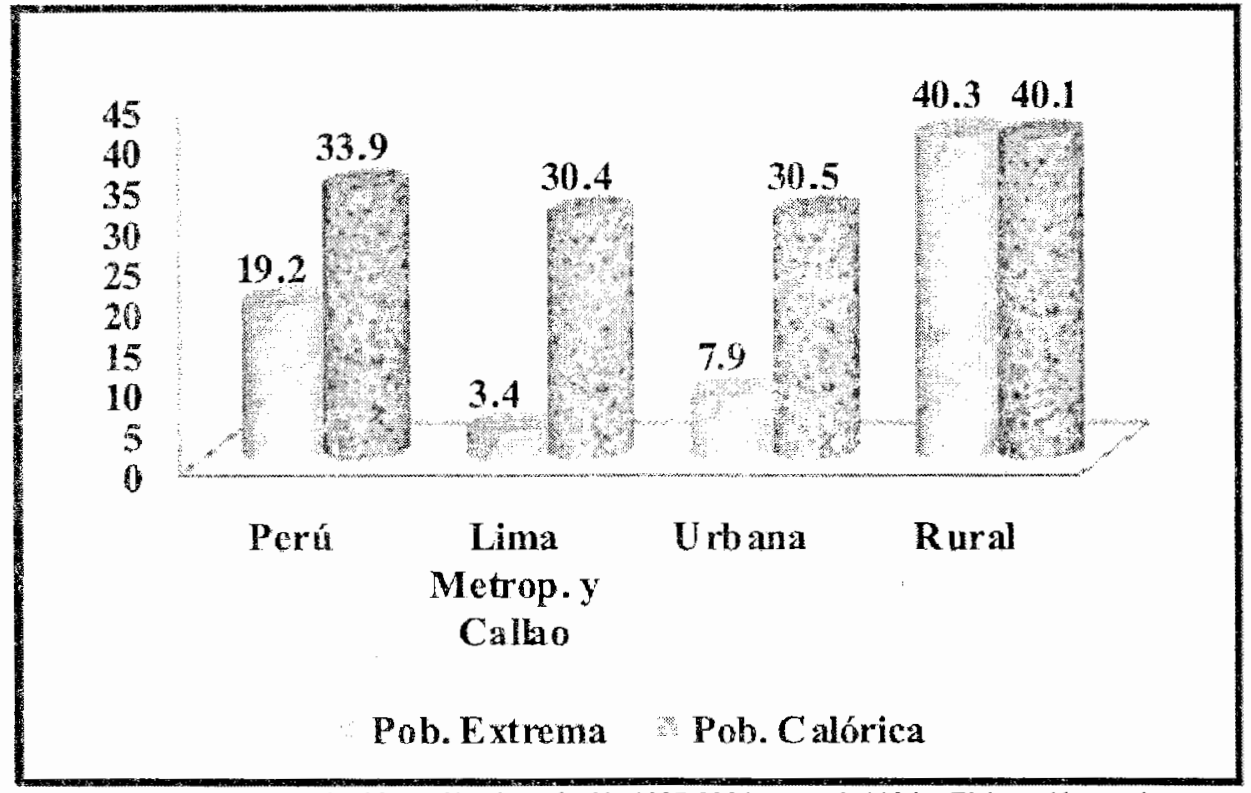

Fucnte: INEI, Condiciones de vida en el Perú, evolución 1997-2004. Mayo de 2006. Elaboración propia

La PE mide el gasto per cápita en relación al costo de la CBA, la PC mide el gasto en calorías en relación al requerimiento calórico. Tengamos en cuenta que la cantidad de calorías compradas no es lo mismo que la cantidad de calorías consumidas. Consecuentemente, lo más probable es que el déficit real de calorías en relación al requerimiento calórico sea mayor que el que las estadísticas registran. Otra mirada conduce a pensar en desequilibrios al interior del hogar, ya que los cálculos per cápita, a partír de los hogares, encubren desigualdades reales.

La PC es consecuencia, en primer lugar, de que el ingreso que se convierte en gasto es inferior al necesario para adquirir la CBA; $y$, en segundo lugar, los ingresos que se encuentran por encima de la CBA no se orientan adecuadamente para cubrir los requerimientos de nutrientes.

Durante el prirner quinquenio del siglo XXI la PC ha crecido de manera sostenida en las poblaciones urbanas y en Lima Metropolitana y el Cailao en particular, como se ve en el gráfico 3. La alimentación está relacionada directamente con la nutrición, ésta a la salud y ambas a la educación. Si la tercera parte de la población urbana incluida Lima Metropolitana y el Callao sufre PC, la situación es gravísima. 
Pobreza y desnutrición en el perú: explorando la última década

Gráfico $N^{\circ}$ 3: Perú: Evolución de la pobreza calórica total y por área, 2001-2004. (cifras relativas)

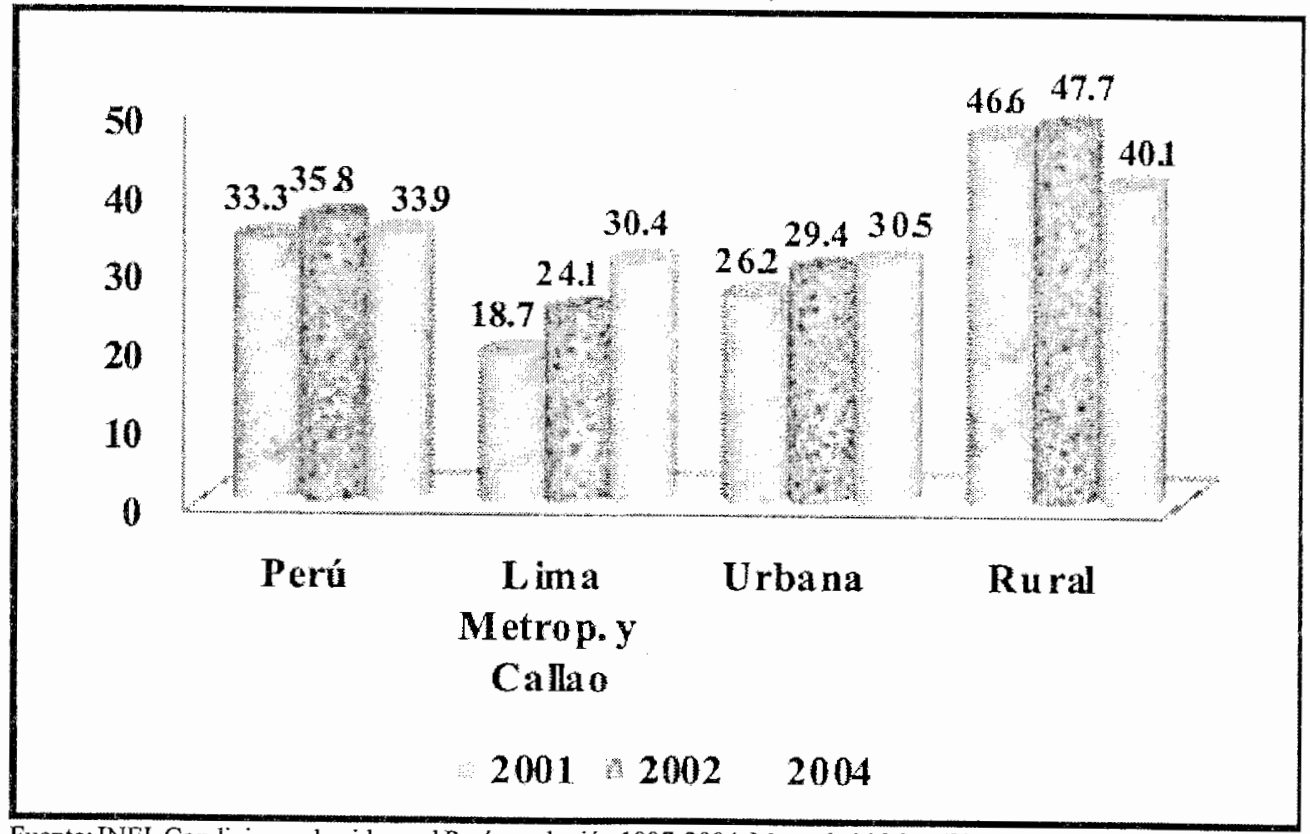

Fuente: INEI, Condiciones de vida en el Perú, evolución 1997-2004, Mayo de $2006 . \quad$ Elaboración propia

El PBI durante los últimos años ha crecido a tasas de 5\%,6\% y $7 \%$, las exportaciones también han crecido de manera significativa, en pocos años han logrado duplicarse, pero paralelamente ha crecido la PC sobre todo en la ciudad capital y el primer puerto.

Uno de cada tres peruanos tiene déficit energético, son vulnerables a las enfermedades que con el transcurrir del tiempo dirán presente, alterando el normal desempeño actual o futuro de sus actividades, en cualesquiera de las distintas etapas de la vida por la que estén transitando. El modelo no contribuye a gestar y mejorar el capital social en el país. Cómo pensar en una población emprendedora si las capacidades, destrezas, habilidades y potencialidades han sido pasmadas. En todo caso hay un amplio contingente de personas a las que la igualdad de oportunidades le será esquiva.

Finalmente, el gráfico 4 presenta una vista panorámica de la desnutrición crónica de los 


\section{Luis Reyes Lostaunau}

niños menores de 5 años desde 1991-92 hasta el 2004-05. A nivel nacional y rural la caída de la desnutrición crónica fue importante durante la primera mitad de la década de los noventa, luego en ambos casos se estableció el estancamiento. En las urbes la caída fue sostenida.

De los gráficos 3 y 4 podemos afirmar que en las ciudades la desnutrición crónica en niños menores de 5 años se ha reducido con el paso del tiempo, en cambio la pobreza calórica aumentó. Es probable que ello responda a los programas sociales alimentarios focalizados, centralmente, en los niños citadinos de la edad en mención. En cambio con la población en general se aplica la política de sálvese quien pueda, y un gran grupo no puede hacerlo.

\section{Gráfico $\mathrm{N}^{\circ} 4$ : Perú :}

Evolución de la desnutrición crónica por áreas en niños menores de 5 años (1991-1992) - (2004-2005), cifras relativas.

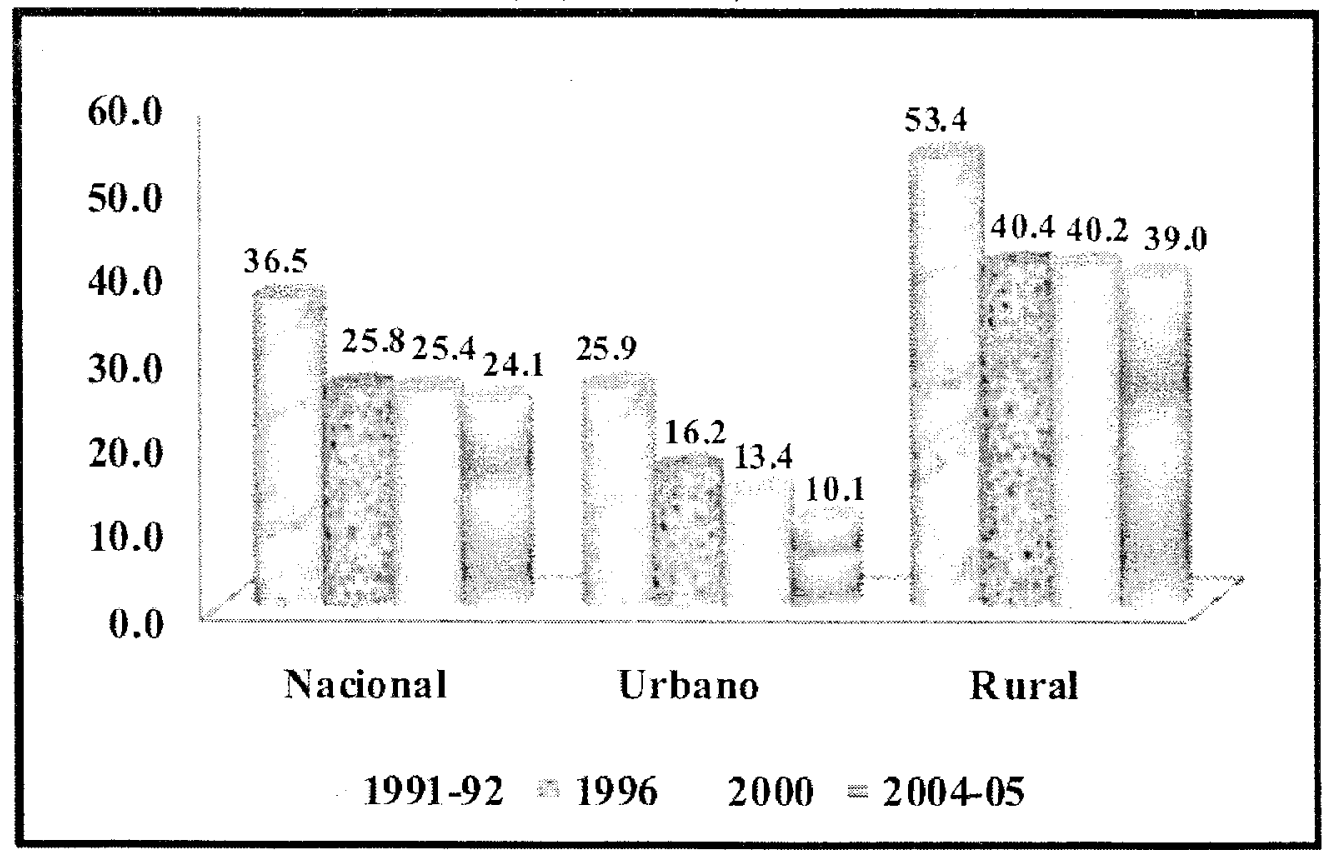

Fuente: INEI, USAID, MEASURE DHS. ENDES Continua 2004-2005. Junio 2006. 
Pobreza y desnutrición en el perú: explorando la última década

\section{A modo de conclusión.}

El concepto de pobreza calórica desplaza del centro de la lucha contra la pobreza a la pobreza extrema porque tiene una dimensión más completa que trasciende lo estrictamente monetario. Es más, al compararlas, hasta tienen comportamientos disímiles, encontramos que sus tendencias han sido opuestas en el caso de las urbes. A pesar de haber decrecido la tasa de pobreza extrema, lo contrario ha ocurrido con la tasa de pobreza calórica, lo que le quita importancia a la primera porque su referente es la Canasta Mínima Alimentaria y no los requerimientos nutricionales de las personas, que son quienes importan

La lucha contra la pobreza y la desnutrición no puede ser la lucha contra exigencias mínimas para mejorar las estadísticas, sino contra exigencias que permitan que las personas se realicen y el país se incorpore a la senda del desarrollo.

\section{BIBLIOGRAFÍA}

- INEI. Condiciones de vida en el Perú: Evolución 1997-2004. Lima, mayo de 2006.

- INEI-USAID-MEASURE DHS. ENDES Continua 2004-2005. Lima, junio de 2006.

- Restrepo, María Teresa. Estado nutricional y crecimiento físico. Editorial Universidad de Antioquia. Medellín, setiembre de 2000. 